Explorer) satellite team led by George Smoot crowned these ground-based achievements by detecting minute imprints on the cosmic microwave background, at a level and on a scale generally consistent with both the CDM prediction and the reality of the large-scale structure and the large-scale flows.

I say "generally consistent" because the past three years have seen considerable wrangling over whether the diverse parts of the cosmology puzzle truly fit together or whether additional work with a theoretical hacksaw and file is necessary. Dressler takes the moderate, and I think correct, view that the story so far is remarkable for its success, even if it is not yet complete. Indeed, right now, both theorists and observers are filing away at a poor fit between the value of the Hubble constant, the apparent age of globular cluster stars and the continuing belief (and some evidence from the large-scale flows) that $\Omega=1$.

Although he may never again have a story to tell with a plot as compelling as his own adventures with the six other Samurai, one hopes that Dressler will continue in further charming books to recount the saga of modern cosmological discovery as it continues to unfold.

William $H$. Press is at the Harvard-Smithsonian Center for Astrophysics, 60 Garden Street, Cambridge, Massachusetts 02138, USA.

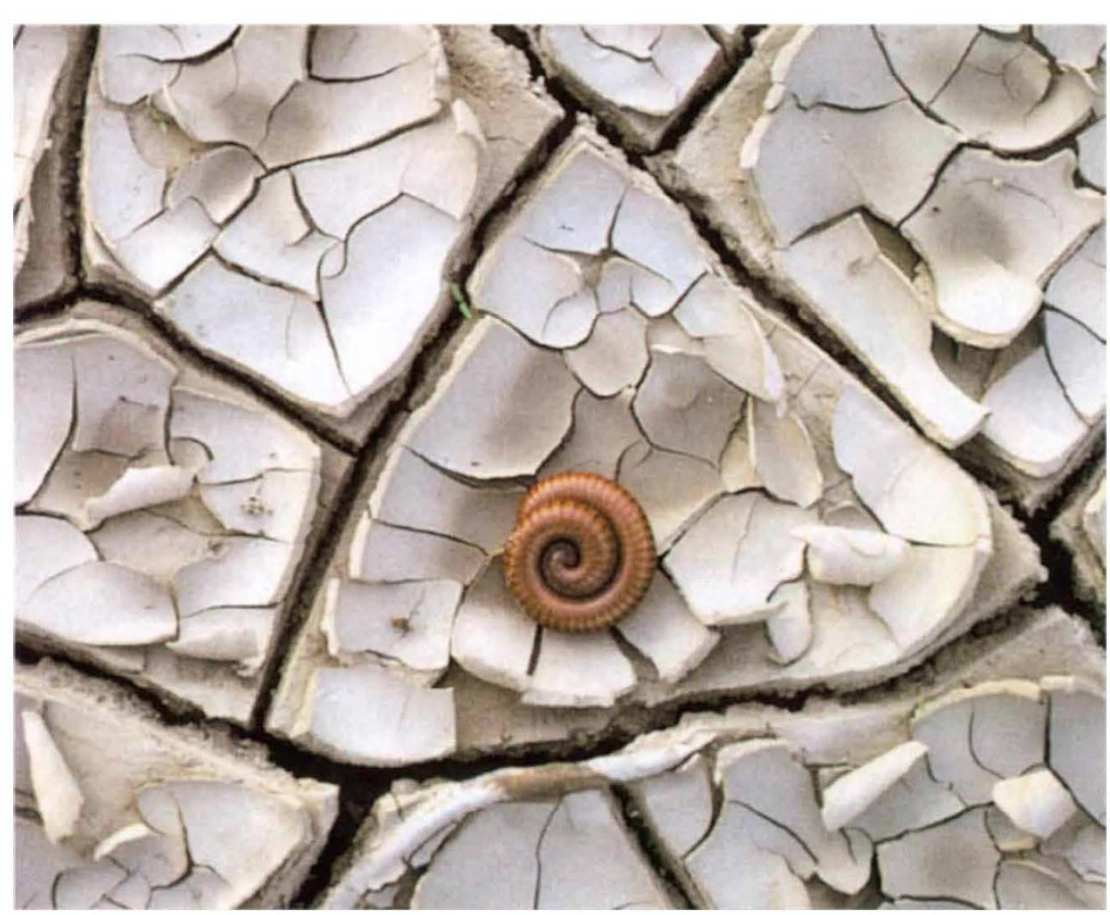

A DESERT millipede Orthoporus ornatus on scrubland near the Rio Bravo in Coahuila Mexico. This picture, and the one on the facing page, are taken from Two Eagles/Dos Aguilas, a magnificent photographic celebration of the natural world of the United States/Mexico borderlands. Published by University of California Press in association with the Nature Conservancy, $\$ 55$.

\section{Drag artist}

\section{Rory Howlett}

Life in Moving Fluids: The Physical Biology of Flow, Second Edition. By Steven Vogel. Princeton University Press: 1994. Pp. 467. \$49.50, £35.

FORM and function are the meat and drink of comparative biology, but a whole generation of factory-trained molecular technicians are poorly equipped to appreciate the morphological subtleties of the animals and plants they purport to study. Ask the average Drosophila developmental geneticist why his or her beloved fruitflies devote just half of one per cent of their body mass to wings and you are unlikely to be any the wiser after hearing the answer. Those who wish to know the answer to this and a vast array of related questions should turn to this fascinating book - now in its second edition - in which Steven Vogel, a biologist in the true sense of the word, explores the sometimes turbulent interface between fluid dynamics and biology, with examples ranging from bacteria to brachiopods, from kelp to killer bees.

The subject of the book forms a branch of biophysics in which the polymerase chain reaction is about as useful as a patent office would be to a human geneticist with a belief in the sanctity of the

genome. It is a field where researchers build ingenious devices for the measurement of tiny pressure differentials in inaccessible places, minimum and maximum flow velocities, drag coefficients and the like. Moreover, it is fun: stuffed birds sprayed with hairspray are mounted in wind tunnels to study the effect of feather fluttering on drag and lift; scale models of prairie dog burrows are built to ventilate questions of passive airflow; penguin carcasses are dragged at various speeds behind motor launches to measure drag, with and without wings; a glass tube with an enlargement just upstream of the final orifice is used to illustrate how both the human penis and ink-jet printers are designed to minimize the formation of those inconvenient 'satellite droplets'.

The last example highlights the analogies that can often be drawn between the functional morphology of organisms and the design of human-built contraptions: engineering considerations and physical concepts are to the fore - and it is here that the typically innumerate biologist waves his or her arms in horror and retreats to more qualitative territories. Much of the value of Vogel's book is that it provides a clear and accessible account of physical measurement, theory and analysis before diving into the detailed intricacies of specific biological examples. Starting with the relatively simple examples that assume laminar, nonturbulent flow, he guides the reader through the basic principles of fluid dynamics, including streamlines, continuity of flow, the role of pressure and momentum, surface effects and velocity gradients.

Throughout, attention is drawn to the sometimes paradoxical behaviour of fluids and to the importance of the many dimensionless ratios, such as the ubiquitous Reynolds number, describing the relative importance of inertial and viscous forces in any given biological situation, whether it be the flow of blood through the vascular system of vertebrates or the dispersal of fungal spores. More complex phenomena such as turbulence and vortex shedding are handled deftly, leaving the reader confident that he or she need not despair even in the face of biological problems characterized by complex, nonsteady flows.

All in all, this is a fine book, with an extensive up-to-date reference list, and written with enthusiasm and humour. It is heartily recommended to all serious students of biology, and especially to those who sympathize with Vogel's sentiment, aired as a coda, that although we may live in an age of great advance in the biological sciences, progress per investigator may be at a low point, with faddishness to blame.

Rory Howlett is an associate editor of Nature. 